\title{
An Adaptive Method Using Genetic Fuzzy System to Evaluate Suspended Particulates Matters SPM from Landsat and Modis Data
}

\author{
Bahia Lounis, Sofiane Rabia, Adlene Ramoul, and Aichouche Belhadj Aissa \\ Laboratory of image processing and radiation- Faculty of Electronics and Computer Science- \\ USTHB University - BP 32, El-Alia-Beb-Ezzouar 16111-Algiers-Algeria \\ lounisbahia@yahoo.fr, h.belhadj@mailcity.com
}

\begin{abstract}
In this paper, we propose an optimization of fuzzy model which exploits remotely sensed multispectral reflectances to estimate Suspended Particulates Matters SPM concentrations in coastal waters. The relation between the SPM concentrations and the subsurface reflectances is modeled by a set of fuzzy rules extracted automatically from the data through two steps procedure. First, fuzzy rules are generated by unsupervised fuzzy clustering of the input data. In the second step, a genetic algorithm is applied to optimize the rules. Our contribution has focused on global and partial optimization of rules and a proposed chromosome structure adapted to remote sensing data. Results of the application of each type of optimization to Landsat and Modis data are shown and discussed.
\end{abstract}

Keywords: Remote sensing data, Coastal water quality, SPM concentrations, Fuzzy clustering, Genetic optimization, Chromosome codification.

\section{Introduction}

In major coastal waters, Suspended Particulate Matters "SPM" is the biggest source of water pollution. The determination of their concentration is basic to the study of environmental phenomenology and manage the coastal environments. Furthermore, the SPM is, also, essential in the initialization and validation of numerical hydrosedimentary models [1], and is considered as an important indicator of erosion problems in the watershed. For these reasons, the concentration of this parameter must be continuously monitored in order to follow their seasonal movements, to quantify sedimentary fluxes and estimate the fluvial solid discharges to the ocean. To reach this goal, spatial and/or aerial remote sensing is the most efficient tool for mapping the concentration of SPM as it provides an instantaneous and synoptic view of sediments that would otherwise be unavailable by in situ measurements.

SPM concentrations affect remotely sensed signals through the interactions of scattering and absorption by sediment particles and water. Summaries of these relationships are provided in [2]. In general, optics of water-sediment mixtures are highly nonlinear, while many factors such as suspended particle size, shape and color can have large influences on water-sediment optics. Due to these complexities it is 
well known that there are no universal algorithms to remotely estimate sediment concentrations and the most first developed algorithms were both empirical or semi analytical models [3]. With the increase of multi-spectral information and the complexity to solve the inverse model, these last years, new estimation algorithms inspired from natural phenomena have emerged. Neural networks were successfully used to implement the inverse model and to properly address the non-linearity problem [4]. Besides neural networks, fuzzy systems have proved to be particularly effective in identifying non-linear models too. The most popular approach to fuzzy modeling is based on the identification of fuzzy rules, which describe in linguistic terms the input/output relationship [5]. These rules are extracted from the available numerical data and fixe the actions that must be performed to obtain the output if some conditions on the input variables are satisfied. The crucial point of this method is the definition of membership functions which express the membership degrees of the input variables to their appropriate fuzzy sets. An optimization process is usually needed to tune membership functions so that the fuzzy method implements the desired inverse model.

In this paper, we propose a methodological approach for SPM estimation based on the hybridization of fuzzy logic and genetic algorithms (GAs) using Landsat and Modis data. In this approach, we devote the first part of it to the fuzzy SPM estimation based on the fuzzy rules of Takagi Seguno kang TSK type[5]. We establish the membership functions of the fuzzy sets, which are the most important parameter of SPM estimation model. We then present, in the second part of our approach, the optimization process in which we involve a TSK population to generate an accurate membership functions and TSK rules. Our contribution consists in the search of the best optimization that gives the best estimation results. We then perform a global and partial optimization by adapting the chromosome structure codifying fuzzy sets parameters. Further details of these two parts are presented in section three and four. In the experimental section, we present the estimation results and we show the benefits of each optimization type.

\section{Processed Data}

To carry out our experiments, we have used two images with different spatial resolution, $30 \mathrm{~m}$ and $1 \mathrm{~km}$, corresponding respectively to ETM+ and Modis satellite and covering the Algiers bay and the Manche Sea. The ETM image (1342x489 pixels) has been, first, geometrically corrected, georeferenced and transformed into radiances. After, it was corrected from atmospheric contribution by the dark point technique [6] and masked from the land areas. Whereas the Modis image (1667x2401 pixels) has been provided with all necessary pretreatments. From these images and according to the available concentrations of SPM, we have built two data sets of subsurface reflectances $R$ and $S P M$ concentrations, 282 and 7093 pairs of $\left(R_{j}, S P M\right)$ For ETM and Modis respectively. For each set, we randomly split the data into two subsets, the former subset composed of the $2 / 3$ of the initial data is used to define the fuzzy model whereas the latter to evaluate its performances. 


\section{Fuzzy SPM Estimation}

The main idea of fuzzy SPM estimation is based on unsupervised fuzzy clustering of the available data to clusters in order to select a blend water types. Each cluster $i$, which shares the same spectral characteristics, is associated to a water type. For each water type, the SPM concentration variation is assumed to be locally linear and expressed as following: $S P M_{i}=p_{0, i}+p_{i, 1} R_{1}+p_{i, 2} R_{2}+\ldots+p_{i, M} R_{M}$, where $R_{M}$ are the $M$ surface reflectances and $\left(p_{i, 0}, p_{i, 1}, p_{i, 2}, \ldots, p_{i, M}\right)$ are real numbers. The estimation model is, then, expressed as a set of conditions, which assign each pixel to a water type under the form of Takagi-Sugeno-Kang (TSK) rules. The premises of these rules depend on the fuzzy sets $A_{i, j}$ defined on the reflectance domain and the consequences present the local linear SPM model form, like shown by equation 1[5].

$$
\text { Rule }_{i} \text { : If }\left(\mathrm{R}_{1} \text { is } \mathrm{A}_{\mathrm{i}, 1}\right) \& \ldots \&\left(\mathrm{R}_{\mathrm{M}} \text { is } \mathrm{A}_{\mathrm{i}, \mathrm{M}}\right) \text { Then } \mathrm{SPM}_{\mathrm{i}}=\mathrm{p}_{\mathrm{i}, 0}+\mathrm{p}_{i, 1} \mathrm{R}_{1}+\ldots+\mathrm{p}_{\mathrm{i}, \mathrm{M}} \mathrm{R}_{\mathrm{M}}
$$

Each local model is associated with a degree of activation $\beta_{i}$ expressed as $\beta_{i}=\min \left(u_{A_{i, 1}}\left(R_{1}\right), u_{A_{i, 2}}\left(R_{2}\right), \ldots, u_{A_{i, M}}\left(R_{M}\right)\right)$, where $u_{A_{i, j}}\left(R_{j}\right)$ is the membership function associated to the fuzzy set $A_{i, j}$. The global SPM concentration is, then, computed by aggregating the conclusions inferred from $r$ individual rules as followed:

$$
S P M=\frac{\sum_{i=1}^{r} \beta_{i} \cdot S P M_{i}}{\sum_{i=1}^{r} \beta_{i}}
$$

Consequently, the estimation model is regarded as an identification problem of fuzzy sets $A_{i, j}$ and their membership functions $u\left(A_{i, j}\right)$, which define the different water types, and parameters identification $\left(p_{i, 0}, p_{i, 1}, p_{i, 2}, \ldots, p_{i, M}\right)$ of linear model associated to each cluster. All these parameters are extracted from an unsupervised partition of the available data using fuzzy clustering algorithm.

Let $Z=\left\{z_{1}, z_{2}, \ldots, z_{N}\right\}$ be a training data set, with $z_{k}=\left(R_{k}, S P M_{k}\right)$, where $R_{k}$ is the $\mathrm{k}^{\text {th }}$ vector of subsurface spectral reflectances and $S P M_{k}$ is its corresponding suspended particulate matters concentration. Let $C_{1}, \ldots, C_{C}$ be a family of fuzzy clusters on $Z$, and $U_{C, N}$ is a real $[C x N]$ matrix. A fuzzy C-partitions of $Z$ is the construction of the matrix $U_{C, N}$ as $U=\left\{U \mid u_{i, k} \in[0,1] \forall i ; \sum_{i=1}^{c} u_{i, k}=1 \forall k ; 0<\sum_{k=1}^{N} u_{i, k}<N \forall i\right\}$ where $u_{i, k}$ is the membership value of $z_{k}$ to a cluster $C_{i}$. The FCM algorithm determines the optimal fuzzy C-partitions minimizing the objective function $J_{m}(U, V, W)=\sum_{i=1}^{c} \sum_{k=1}^{N} u_{i, k}^{m} d_{i}^{2}\left(z_{k}, v_{i}\right)$ where $d_{i}^{2}\left(z_{k}, v_{i}\right)=\left(z_{k}, v_{i}\right)^{T} W_{i}\left(z_{k}, v_{i}\right)$ and $m$ is the fuzzification coefficient. Each cluster $C_{i}$ is represented by a prototype $v_{i}$ in $\Re^{M}$ and 
its matrix $W_{i}$ which determines its shape. In the classical FCM algorithm, $W_{i}$ is an identity matrix and the distance is Euclidian [7]. In Gustafson and Kessel algorithm [8], known as GK algorithm, $W_{i}=\left[\rho_{i} \operatorname{det}\left(Q_{i}\right)\right]^{1 / c} Q_{i}^{-1}$ where $Q_{i}$ is the fuzzy covariance matrix of cluster $i$ and the clusters are hyper-ellipsoidals. Whereas in [5], noted Weighted fuzzy C-means (WFCM) algorithm, $W_{i}$ is forced to be diagonal whose diagonal elements are $w_{i, f, f}$. This latest is equivalent to use a weighted distance, where each element $w_{f, f}$ on the diagonal of $W_{i}$, weights the importance of feature $f$ in computing $d_{i}$. Comparing to classical FCM and GK algorithms, the WFCM presents simplicity of implementation, rapid convergence and best performances [5]. That's why we have chosen it to partition the input space for our data. The WFCM principle is summarized as followed, fix the number of clusters $C$, the fuzzification coefficient $m$, the weight $h$ and initialize $U_{C, N}^{(l=0)}$ randomly. Then, at the step $l=0,1,2, \ldots$. do:

1. Calculate the vector prototypes $V^{(l)}$ using $U_{C, N}^{(l)}$,

2. Calculate $W_{i}^{(l)}$ using $V^{(l)}$ and $U_{C, N}^{(l)}$,

3. Update $U_{C, N}^{(l+1)}$ using $V^{(l)}$ and $W_{i}^{(l)}$

4. Compare $U_{C, N}^{(l)}$ and $U_{C, N}^{(l+1)}$ using the matrix norm: $\left\|U_{C, N}^{(l+1)}-U_{C, N}^{(l)}\right\|$, if $\left\|U_{C, N}^{(l+1)}-U_{C, N}^{(l)}\right\| \leq \varepsilon$ then stop otherwise go to 1 , with $l=l+1$ and $\varepsilon$ is the admissible error generally $\varepsilon=10^{-5}$.

Once the input space is partitioned, each cluster is associated to a fuzzy set $A_{i, j}$. The membership functions $u_{A_{i, j}}$ of $A_{i, j}$ are obtained by projecting the rows of the partition matrix $U_{C, N}$ onto the input variables $R_{j}$ and approximating the projections by triangular membership functions as expressed in equation 3.

$$
u_{A_{i, j}}\left(R_{j}\right)=\max \left(0, \min \left(\frac{R_{j}-a}{b-a}, \frac{c-R_{j}}{c-b}\right)\right)
$$

Where $b$ corresponds to the abscissa of the vertex of the triangle and is computed as the weighted average of the components of the training patterns. Parameters $a$ and $c$ are deduced as the intersection of the abscissas axis with the lines obtained as linear regression of the membership values of the training patterns, respectively, on the left and the right sides of $b$. Given the membership functions, the parameter $\left(p_{i, 0}, p_{i, 1}, p_{i, 2}, \ldots, p_{i, M}\right)$ of local linear model associated to each cluster are carried out by standard linear least-squares methods as described in [9].

$$
\mathrm{P}=\left(\mathrm{X}^{\mathrm{T}} \mathrm{X}\right)^{-1} \cdot \mathrm{X}^{\mathrm{T}} \cdot \mathrm{Y}
$$




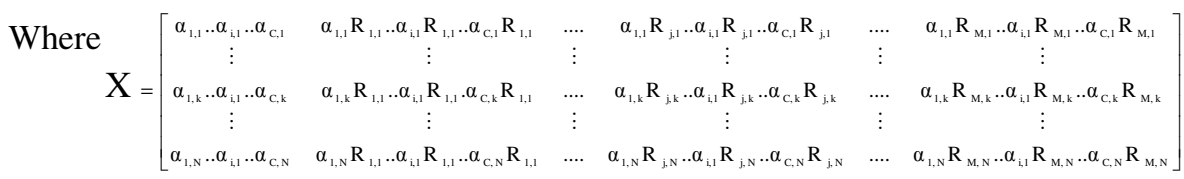

$$
\begin{aligned}
& \mathrm{P}=\left[\mathrm{p}_{1,0}, \ldots \ldots . \mathrm{p}_{1, \mathrm{M}}, \ldots \ldots \ldots . . . \mathrm{p}_{\mathrm{c}, 0}, \ldots \ldots ., \mathrm{p}_{\mathrm{c}, \mathrm{M}}\right], \mathrm{Y}=\left[\mathrm{SPM}_{1}, \ldots \ldots \ldots . \mathrm{SPM}_{\mathrm{k}}\right]^{\mathrm{T}}
\end{aligned}
$$

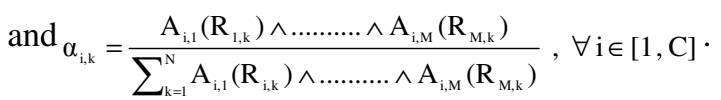

After identifying all fuzzy rules, a genetic optimization is applied in order to tune their parameters and reach the desired estimation results.

\section{Genetic Optimization of Fuzzy Model}

Genetic Algorithms (GAs) are adaptive methods suitable to solve optimization problems. By mimicking the principles of natural selection, GAs are able to evolve solutions for real world problems provided that they have been suitably encoded. Each potential solution to a given problem is coded as a binary or real string that is called chromosome. Generally, GAs start with a randomly generated initial population of chromosomes. At each step, a new population is generated from the current one using two basic operators: crossover and mutation. While crossover combines parent chromosomes to generate offspring chromosomes, mutation is a local modification of a chromosome. Chromosomes are selected for crossover and/or mutation based on their fitness function.

In our model, the general objective of the genetic optimization is to find a fuzzy model which can approximate input/output relationship by optimizing the structure of the model and/or it's parameters. As cited above, the fuzzy SPM estimation model is based on a number of rules, which are defined by the fuzzy sets and output parameters extracted from the partition of the input space. Several techniques are available to optimize the structure of fuzzy models. According to the model complexity, these techniques proceed by global or partial optimization taking account all or one part of the fuzzy model. To adapt the GA to a specific optimization, the chromosome structure is adjusted to the parameters which are tuned.

\subsection{Structure Codifying Chromosome}

In a global optimization, each chromosome represents the entire fuzzy system, ruleby-rule, with the antecedent and consequent parts. As shown by figure 1.a, each rule antecedent consists of a sequence of $M$ triplets $(a, b, c)$ of real numbers representing triangular membership functions, whereas each rule consequent contains $M+1$ real numbers corresponding to the consequent parameters $P_{i, j}$ (see section 3 ). As the identification of the antecedent and consequent parts of a rule are inseparable since they have a mutual relationship. Thus, the optimization of parameters needs much more processing time to reach desired estimation results. For some applications, the GA is adapted to a specific problem, each chromosome then codifies one part of the 
fuzzy model, generally the membership functions. As illustrated by figure 1.b, the chromosome structure is composed of the antecedent part of the rule, which defined the membership functions $(a, b, c)$ of the fuzzy sets.

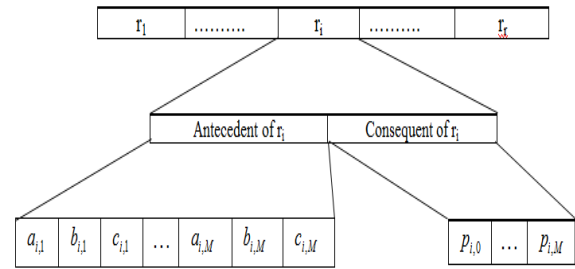

(a)

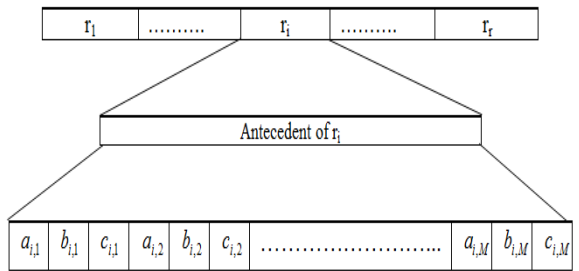

(b)

Fig. 1. Chromosome representation. (a) : Global optimization, (b) : Partial optimization.

In this work, we propose to test both types of optimization in order to select the best one for our databases. In fact, the complexity of the inverse model of SPM concentration depends on several parameters such as the atmospheric and geographic conditions specific to the studied area, its suspended particle size, shape, and color....

\subsection{Genetic Evolution}

For each optimization type (global or partial), the most important consideration in the implementation is to define a proper fitness function which aids to find a better solution for the given optimization and the AG evolution which ensure the algorithm convergence. As we are interested by the generation of the SPM concentrations with minimizing the error estimation, we defined the fitness value as the inverse of the Mean Square Error MSE. Also, to accelerate the convergence, fuzzy parameters resulting from the partition algorithm are considered as the initial population of the AG and we apply the arithmetic crossover and the uniform mutation operators to generate a new population [10]. Chromosomes to be mated are chosen by using the well-known roulette wheel selection method, which associates to each chromosome a probability proportional to its fitness value. We fixed the probability of crossover and mutation to 0.9 and 0.1 , respectively. The offspring generated using crossover and mutation are checked against the aforementioned space coverage criterion. Only $80 \%$ of the new population is composed of offspring, whereas $20 \%$ consists of the best chromosomes of the previous population. When the average of the fitness values of all the individuals in the population is greater than $99 \%$ of the fitness value of the best individual or a prefixed number of iterations has been executed, the GA is considered to have converged.

\section{Experimental Results}

To estimate the SPM concentrations for ETM and Modis data sets, we proceed, first, to the partition of both data by executing the clustering with the Weighted Fuzzy C-means (WFCM) algorithms proposed in [5] in order to deduce the fuzzy sets 


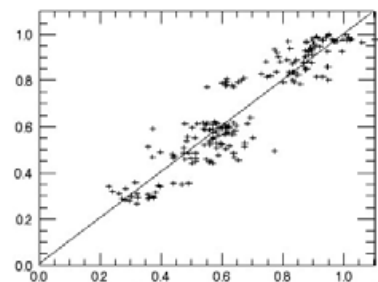

(a)

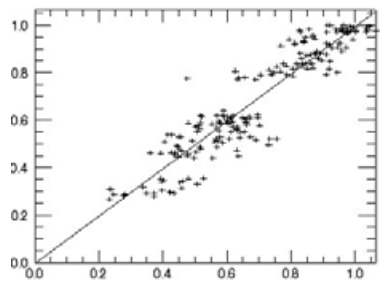

(b)

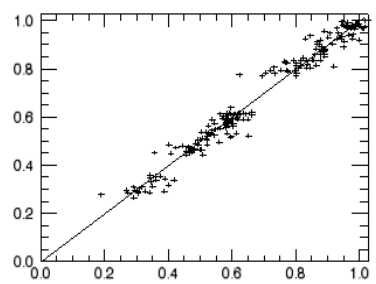

(c)

Fig. 2. Fuzzy model performances for training base data of ETM. (a) : Fuzzy model, (b) : Fuzzy with global optimization (c) : Fuzzy with partial optimization.

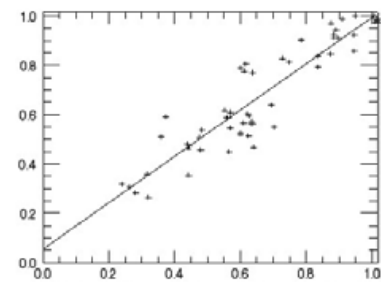

(a)

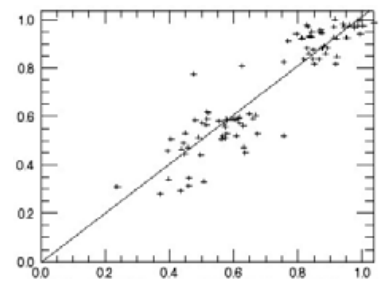

(b)

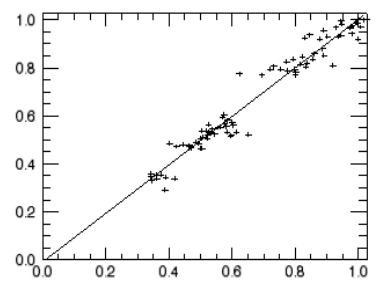

(c)

Fig. 3. Fuzzy model performances for test base data of ETM. (a) : Fuzzy model, (b) : Fuzzy with global optimization (c) : Fuzzy with partial optimization.

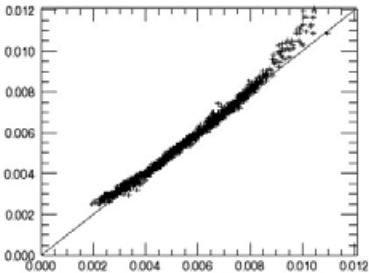

(a)

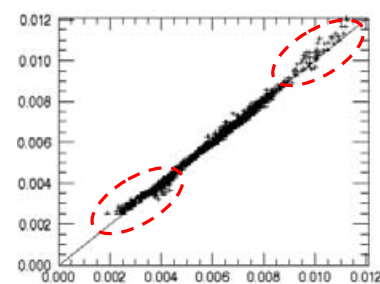

(b)

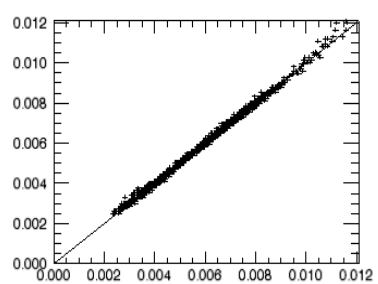

(c)

Fig. 4. Fuzzy model performances for training base data of Modis. (a) : Fuzzy model, (b) : Fuzzy with global optimization (c) : Fuzzy with partial optimization.

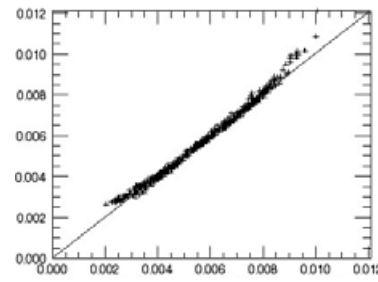

(a)

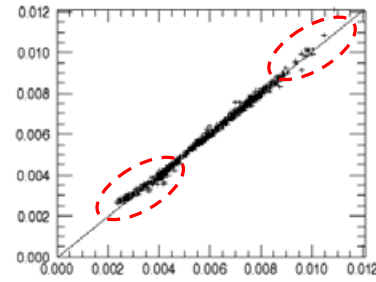

(b)

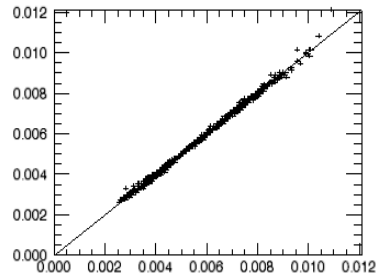

(c)

Fig. 5. Fuzzy model performances for test base data of Modis. (a) : Fuzzy model, (b) : Fuzzy with global optimization (c) : Fuzzy with partial optimization. 
Table 1. Statistic comparison of fuzzy model performances before and after optimization

\begin{tabular}{|c|c|c|c|c|c|c|c|}
\hline & \multicolumn{2}{|c|}{ Fuzzy model } & \multicolumn{2}{|c|}{ Global optimization } & \multicolumn{2}{|c|}{ Partial optimization } \\
\hline & & $M S E$ & $\rho$ & $M S E$ & $\rho$ & $M S E$ & $\rho$ \\
\hline \multirow{2}{*}{$\sum_{\text {至 }}$} & Training data & 0,0081039 & 0,9142 & 0,0065202 & 0,9313 & 0.0015308 & 0.9842 \\
\hline & Test data & 0,0084515 & 0,9093 & 0,0074373 & 0,9212 & 0.0021842 & 0.9762 \\
\hline \multirow{2}{*}{ 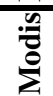 } & Training data & $1,93138 \mathrm{e}^{-8}$ & 0,9952 & $9,57956 \mathrm{e}^{-9}$ & 0,9976 & $4.68912 \mathrm{e}^{-9}$ & 0.9988 \\
\hline & Test data & $2,33829 \mathrm{e}^{-8}$ & 0,9954 & $1,23177 \mathrm{e}^{-8}$ & 0,9976 & $6.70072 \mathrm{e}^{-9}$ & 0.9987 \\
\hline
\end{tabular}

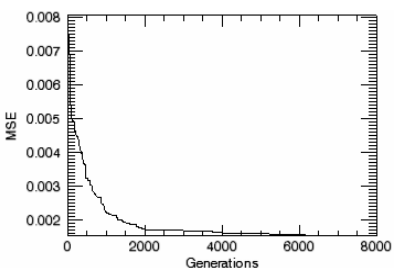

(a)

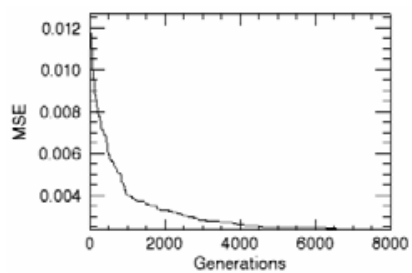

(b)

Fig. 6. MSE variation versus iteration number. (a) ETM data (b). Modis data.

parameters $\left(A_{i, j}, u\left(A_{i, j}\right)\right)$, which define the different water types, and the associated output parameters $\left(p_{i, 0}, p_{i, 1}, p_{i, 2}, \ldots, p_{i, M}\right)$. Then, we computed the SPM concentrations and evaluated the performances of the fuzzy model by calculating the mean square error MSE and the correlation coefficient $\rho$ of each type of data. An important point in the implementation of fuzzy SPM model is to determine the optimum number of clusters $C$ which determine the number of rules $r$. Like in [5] we have adopted a criterion Xie-Beni index and we have obtained 5 and 9 rules for Modis and ETM respectively. Figures 2.a, 3.a, 4.a and 5.a show the comparison between the fuzzy estimated and the measured values of SPM for ETM and Modis data. For these figures, we note that the values are correlated; they approach to the bisector despite those obtained for ETM data, which present some dispersion. The MSE and the correlation coefficients presented in table 1 confirm these remarks.

Figures 2.b, 3.b, 4.b, 5.b and 2.c, 3.c, 4.c, 5.c illustrate the results of global and partial genetic optimization, respectively. Lets' note that to be able to compare these two types of optimization, we have taken the same hypothesis, to know the initial population, the numbers of iterations, the same crossover and mutation probability, fitness and convergence criteria. Figure 6 illustrates an example of MSE evolution during the global optimization.

Figures 2.b, 3.b, 4.b and 5.b illustrate the results of global genetic optimization. Compared to figures 2.a, 3.a, 4.a and 5.a, these graphs show the contribution of this optimization to improve the estimation results. Indeed, for Modis data the dispersion of points around the bisector decreased especially for areas encircled in the figures 4.b, and 5.b. However, for ETM data the dispersion has slightly reduced. The statistical results in Table 1 confirm this observation. The correlation coefficient for the ETM data is increased from $91 \%$ to $93 \%$. Thus, it appears that the exploration of 
solution space by encoding both the input and output parameters in a fixed architecture are not adapted to these data.

Figures 2.c and 3.c represent the partial optimization results. The graphs show a clear improvement for ETM data. The partial exploration of the solution space and a mutual relationship between the antecedents and the consequences parameters guarantee more improved results than the global optimization proposed in [6]. This observation is confirmed by the statistical evaluation presented in Table 1. Indeed, the correlation coefficient increased from $91 \%$ to $98 \%$ reducing considerably the MSE error. Whereas, the partial optimization has slightly improved the results of MODIS data as shown by the statistical results presented in table 1 and figures 4.c and 5.c.

\section{Conclusion}

For each data types, we have proposed a genetic optimization of fuzzy model to estimation the SPM concentrations from ETM and Modis data. The fuzzy model is based on fuzzy rules automatically extracted from the data using an unsupervised clustering algorithm. The rules composing the fuzzy model allow easy interpretation of input/output relations between surface reflectances and SPM concentrations. Furthermore, the rules number of fuzzy model for ETM data is approximately the double than that obtained for Modis data. This means that the inverse model of SPM for ETM are more complex compared to Modis model. This gives an initial appreciation of the water quality type corresponding to each studied site. Finally, fuzzy rules parameters are, after, tuned by a genetic algorithm in order to minimize the error between desired and predicted outputs. To each data type, we have proposed two chromosome structures to codify the different rules parameters and to optimize the model in global or partial way. From the experimental results we conclude that the global optimization gives satisfactory results for fuzzy model of Modis data. Whereas, the partial optimization is more suitable for a complex inverse model which corresponds to ETM data.

The generalization of the proposed approach to the entire images provides a map which represents the spatial variability of SPM in the coastal and marine areas. Using the multitemporal remote sensing images, the estimated maps give important information about the SPM spreading. This is allowed to determine seasonal and yearly changes caused for example by floods. These considerations are integrated into our future works.

\section{References}

1. Douillet, P., Ouillon, S., Cordier, E.: A numerical model for fine suspended transport in the southwest lagoon of new caledonia. Coral Reefs 20, 361-372 (2001)

2. Stumpf, R.P., Pennock, J.R.: Calibration of a general optical equation for remote sensing of suspended sediments in a moderatly turbid estuary. Journal of Geophysical Research 94(C10), 14363-14371 (1989)

3. Acker, J., Ouillon, S., Gould, R., Arnone, R.: Measuring Marine Suspended Sediment Concentrations from Space: History and Potential. In: 8th International Conference on Remote Sensing for Marine and Coastal Environments, Halifax, Canada, May 17-19 (2005) 
4. Djellal, D., Lounis, B., Belhadj-Aissa, A.: Cartographie de la concentration des sédiments côtiers par imagerie satellitaire et étude des lidars bathymétriques. In: Journées d'Animation Scientifique (JAS'09) de l'AUF, Alger (Novembre 2009)

5. Cococcioni, M., Corsini, G., Lazzerini, B., Marcelloni, F.: Approaching the Ocean Color Problem Using Fuzzy Rules. IEEE Transactions On Systems, Man, And Cybernetics-Part B: Cybernetics 34(3) (June 2004)

6. Lounis, B., Belhadj aissa, A., et al.: Processus de correction relative PCR des images satellitaires Landsat multidates. In: SETIT 2005, 3rd International Conference: Sciences of Electronic, Technologies of Information and Telecommunications, Tunisia, March 27-31 (2005)

7. Bezdek, J.C.: Pattern Recognition with Fuzzy Objective Function Algorithms. Plenum, NY (1981)

8. Gustafson, D.E., Kessel, W.C.: Fuzzy clustering with fuzzy covariance matrix. In: Gupta, M.M., Ragade, R.K., Yager, R. (eds.) Advances in Fuzzy Set Theory and Applications, pp. 605-620. North-Holland, Amsterdam (1979)

9. Takagi, T., Sugeno, M.: Fuzzy identification of systems and its application to modeling and control. IEEE Trans. System., Man, Cybern. SMC-15, 116-132 (1985)

10. Wei, W.: Synthèse d'un contrôleur flou par algorithme génétique: application au réglage dynamique des paramètres d'un système. Thèse de doctorat de l'université de Lille 1 (1998) 